\title{
Derrame pleural tras cirugía de fijación espinal en pacientes con lesión medular
} traumática

\author{
F.J. Romero-Ganuza; M.A. Marín-Ruiz y E. Díez de la Lastra-Buigues
}

Unidad de Cuidados Intensivos. Hospital Nacional de Parapléjicos. Toledo

\section{Resumen}

El presente estudio se ha diseñado con la finalidad de determinar la prevalencia del derrame pleural tras la intervención de fijación espinal por vía posterior en pacientes con fractura espinal traumática con lesión subyacente de la médula espinal. Se han incluido 20 pacientes intervenidos a lo largo de dos años. Todos ellos han cursado el postoperatorio en la Unidad de Cuidados Intensivos y se les ha practicado exámenes complementarios de control, que incluye estudio radiológico del tórax. La prevalencia de derrame pleural ha sido del $75 \%$. El momento del diagnóstico ha sido dentro de las primeras $\mathbf{4 8}$ horas tras la intervención. El derrame no muestra predominio de ninguno de los dos lados y en general ha sido de escasa cuantía. Sólo en $\mathbf{4}$ casos se ha precisado su drenaje por ocasionar sintomatología respiratoria restrictiva, y en el resto de los casos la evolución ha sido favorable con medidas conservadoras.

PALABRAS CLAVE: Derrame pleural. Cirugía de fijación espinal. Lesión medular.

Pleural effusion after spinal fixation surgery in patients with traumatic spinal cord injury

\section{Summary}

The present study was designed with to determine the prevalence of pleural effusion after posterior approach for spinal fixation in patients with traumatic spinal fracture with underlying spinal cord lesion. The study included 20 patients over two years. The postoperative monitoring has been done in the Intensive Care Unit and have been practiced complementary examinations control, including chest $x$-ray study. The prevalence of pleural effusion was $75 \%$, the time of diagnosis was within the first $\mathbf{4 8}$ hours after the operation. The pleural effusion does not preference for either side, and has

Recibido: 14-03-08. Aceptado: 2-05-08 generally been small claims. Only 4 patients it has been need drainage due to respiratory symptoms restrictive, and the o utcome have been favourable in rest of the cases at conservative treatment.

KEY WORDS: Pleural effusion. Spinal fixation surgery. Spinal cord injury

\section{Introducción}

En pacientes en estado crítico la presencia de derrame pleural puede empeorar la insuficiencia respiratoria asociada a su patología de base junto con la agresión quirúrgica, sobre todo en pacientes politraumatizados que presentan insuficiencia respiratoria de origen neuromuscular derivada de una lesión en la médula espinal cervical o torácica, o en aquellos que presentan como lesión asociada un traumatismo torácico ${ }^{1,7,16}$.

En el abordaje quirúrgico de la columna dorsal por vía anterior o anterolateral, debido a la apertura de la caja torácica, es común la formación de derrame pleural postquirúrgico, por lo que el procedimiento de rutina incluye el mantener unas horas o días un drenaje pleural para evitar el acúmulo de este derrame, pero cuando el abordaje es posterior y no se interviene sobre las estructuras torácicas respiratorias por lo común no se deja tal drenaje $\mathrm{e}^{4,6}$.

Tras constatar la presencia de derrame pleural en algunos pacientes con lesión medular intervenidos para fijación de la columna dorsal por vía posterior, hemos decidido valorar la presencia de derrame pleural en ellos tras la fijación de la fractura de la columna dorsal o dorsolumbar así como su implicación en la evolución del curso clínico del paciente.

Se han descrito algunos casos aislados de derrame pleural tras la cirugía espinal dorsal o dorsolumbar con abordaje posterolateral debido a causas raras, como fístula de líquido cefalorraquídeo ${ }^{11} \mathrm{o}$ lesión del conducto torácico ${ }^{3}$. El desarrollo de un derrame pleural postquirúrgico en la cirugía de columna con abordaje posterior está descrito en algunos trabajos clásicos como "derrame de origen simpá- 
tico", y se considera de buen pronóstico con tratamiento conservador $^{20}$, pero no conocemos la prevalencia real del derrame postquirúrgico, su historia natural ni su tratamiento.

Por ello nuestros objetivos han sido determinar la prevalencia de derrame pleural tras la cirugía de fijación espinal y fijar la historia natural de estos derrames así como su respuesta al tratamiento.

\section{Material y método}

Hemos revisado las intervenciones para fijación de las fracturas dorsales o dorsolumbares en pacientes con lesión medular traumática realizadas de forma no urgente en nuestro Centro a lo largo de 2006 y 2007, así como las de retirada del material de fijación espinal implantado previamente.

Han ingresado en nuestro Centro 709 nuevos pacientes a lo largo de estos dos años, de los que 612 han sufrido una lesión medular de causa traumática. Todos ellos nos han sido referidos desde otros hospitales tras su atención inicial urgente una vez comprobada la presencia de lesión medular. En la mayoría de los casos han sido intervenidos en el hospital de referencia tanto para descompresión como para fijación espinal o para ambas, pero en algunos casos el traslado precoz del paciente se ha realizado con inmovilización externa siendo intervenido posteriormente en nuestro hospital para su fijación espinal, circunstancia que ha tenido lugar en 20 casos.

Hemos hecho el seguimiento de los pacientes intervenidos a lo largo de dos años, entre enero de 2006 y diciembre de 2007, y así hemos podido valorar 20 casos de pacientes con lesión medular a los que se les ha realizado la fijación quirúrgica de su lesión dorsal o dorsolumbar y de los que poseemos registros suficientes para valorar las complicaciones postquirúrgicas. Asimismo, como grupo control, hemos seguido a los 9 pacientes a quienes se realizó durante el mismo periodo de tiempo la retirada del material de fijación implantado anteriormente en el centro de referencia de donde nos fue trasladado para su rehabilitación una vez estabilizado. En ambos casos el postoperatorio inmediato se ha llevado a cabo en la Unidad de Cuidados Intensivos del hospital, al carecer éste de Unidad de Reanimación, y ha sido controlado por sus intensivistas de plantilla.

En todos los casos la técnica quirúrgica, ya descrita anteriormente ${ }^{18}$, ha consistido en abordaje por la línea media posterior, sin toracocentesis ni exposición intrapleural, con disección de las estructuras adyacentes en el abordaje de las vértebras. Tras la reducción se ha procedido a discectomía en caso de protusión discal hacia el canal medular, y posterior fijación segmentaria mediante tornillos transpediculares o ganchos pediculares bilaterales y su unión mediante barras. En el cierre por planos se dejan drenajes subfasciales, pero no tubos intratorácicos, ya que no se ha intervenido sobre la cavidad intrapleural.

El diagnóstico del derrame pleural se ha llevado a cabo mediante la radiografía simple de tórax realizada en decúbito y valorada al menos por el intensivista encargado del seguimiento del postoperatorio del paciente y de un radiólogo del servicio de Radiodiagnóstico del Hospital. Para su comprobación en caso de duda la radiografía se ha repetido usando una proyección anteroposterior del tórax con el paciente en decúbito lateral del lado que se sospechaba el derrame y con rayo horizontal, técnica que descartaba o confirmaba la presencia de líquido pleural definitivamente. Se ha definido la cantidad de líquido pleural como "pequeña" cuando se estimaba radiológicamente una cantidad inferior a los $500 \mathrm{ml}$, "mediana" cuando se estimaba entre 500 y $1.500 \mathrm{ml}$, y como "grande" cuando se estimaba la existencia de más de $1.500 \mathrm{ml}$. de derrame pleural ${ }^{8,15}$.

El tratamiento estadístico utilizado ha sido la chi cuadrado para comparar distribuciones analógicas. El paquete estadístico utilizado ha sido el G-Stat 2.0.

\section{Resultados}

De los 20 pacientes valorados a quienes se ha realizado cirugía de fijación espinal, 18 (90\%) pertenecían al sexo masculino y 2 al femenino. Sus edades estaban comprendidas entre los 16 y los 55 años, con una edad media de 31,5 años. La causa del traumatismo ha sido en 11 pacientes (55\%) un accidente de tráfico y en los 9 restantes precipitaciones de causa laboral o de otro origen.

La lesión vertebral ha sido múltiple en 16 pacientes y única en los 4 restantes. En todos los casos ha estado involucrada alguna vértebra dorsal. El nivel de lesión medular ha sido cervical en 3 pacientes, comprendido entre las metámeras D1 y D5 en 5, entre las metámeras D6 y D10 en 10 casos y por debajo de D10 en los 2 restantes.

En lo que se refiere a las lesiones asociadas al daño espinomedular, en 8 pacientes no se pusieron en evidencia ninguna de estas lesiones. De los 12 restantes han presentado traumatismo torácico 9 pacientes, reflejado como hemoneumotórax en 8 casos, fracturas costales en 7 y contusión pulmonar en 3 , con diferentes combinaciones de estas manifestaciones. Han presentado otras lesiones asociadas en forma de traumatismo craneoencefálico 5 pacientes, traumatismo facial 2 , traumatismo abdominal 3 , traumatismo de miembros con fractura de sus huesos 4 casos y fractura de clavícula y/o escápula otros 4 casos. Estas complicaciones han sido tratadas en todos los casos durante las primeras fases tras el traumatismo, y el tratamiento incluyó la evacuación del derrame en caso de hemotórax. En lo referente a la presencia de traumatismo torácico, de los 15 pacientes con derrame habían presentado traumatismo torácico $8(53,3 \%)$ y no lo habían presen- 
tado 7 (46,7\%), diferencia no significativa, con $\mathrm{p}=0,19$, lo que descarta que la presencia de derrame se asocie con el traumatismo torácico previo a la intervención más que a la intervención misma.

En los 20 pacientes la intervención quirúrgica persigue la reducción y posterior fijación espinal. En 13 pacientes para ello se han utilizado barras largas, ya que ha involucrado más de tres vértebras. En 5 casos se ha utilizado barras cortas que afectan solamente a dos tres vértebras, y en dos casos se ha utilizado el cerclaje transpedicular o interespinoso con injerto autólogo. Se ha usado el sistema MBA en 4 ocasiones, el Sinergy en 8 , el Legacy en 4 y el Plus en 2. De los 15 pacientes con derrame, en 12 (80\%) se han implantado barras largas, por lesiones vertebrales extensas, y en $3(20 \%)$ barras cortas. Esta diferencia es significativa con $\mathrm{p}=0,03$, por lo que se comprueba que cuando es mayor la manipulación quirúrgica, como es el caso de la implantación de sistemas de fijación más amplios y extensos, es mayor la prevalencia del derrame.

Hemos podido comprobar signos de derrame pleural en 15 de nuestros 20 pacientes (75\%). En todos ellos el procedimiento diagnóstico ha sido la radiografía de tórax. En 2 casos se diagnosticó antes de las 24 horas del final de la cirugía, y en 13 pacientes entre las 24 y 48 horas tras la cirugía. La localización ha sido bilateral en 4 pacientes, limitado al hemitórax derecho en otros 4 e izquierdo en los 7 casos restantes. Según la evaluación radiológica la cantidad de líquido era "pequeña" en 10 casos, "mediana" en 3 y "grande" en dos.

Se ha llevado a cabo toracocentesis evacuadora en 4 casos, en todos ellos debido a la existencia de síntomas de insuficiencia respiratoria restrictiva. La cantidad de líquido evacuada ha estado comprendida entre 650 y 1200 $\mathrm{ml}$, con una cantidad media de $865 \mathrm{ml}$. Sólo en un caso se mantuvo el tubo de drenaje más de 24 horas, pudiendo ser retirado tras la toracocentesis en los otros 3 . En los 4 casos la sintomatología respiratoria mejoró en la evaluación realizada a las 6 horas tras la evacuación del derrame. En 3 casos el aspecto del derrame fue serohemático y sin restos macroscópicos de sangre en el cuarto. Su cultivo fue estéril en los cuatro casos y sus características bioquímicas fueron de exudado en todos ellos. En los casos no evacuados se mantuvo una actitud conservadora y expectante hasta comprobar la reabsorción del derrame.

De los 9 pacientes valorados a quienes se ha llevado a cabo una intervención quirúrgica para la retirada del material, ésta se ha realizado por infección del material en 4 casos y por dolor, deformidad o deterioro del material en los otros 5 casos. Sólo hemos podido comprobar entre ellos un caso de derrame pleural postoperatorio, de cantidad pequeña, de localización izquierda y que no precisó su evacuación.

La diferencia en la frecuencia de presentación de derrame pleural en la cirugía de fijación espinal dorsal (75\%) y la de retirada de material de fijación (11\%), realizadas ambas por abordaje posterior, es significativa, con $\mathrm{p}<0,001$.

\section{Discusión}

El desarrollo de un derrame pleural es muy frecuente en una serie de procedimientos quirúrgicos, sobre todo los que utilizan la toracotomía como abordaje y los que invaden la cavidad pleural en cualquiera de los momentos quirúrgicos, así como en otros que no abordan directamente la pleura pero pueden afectar a órganos que están en contacto con ella, como algunos procedimientos abdominales, sobre todo los que intervienen sobre órganos o vísceras del piso abdominal superior'. En general no se advierte de la posibilidad de desarrollar derrame pleural en las intervenciones quirúrgicas para fijación de fracturas de la columna dorsal o dorsolumbar ${ }^{9}$, ni en comunicaciones de experiencias de la cirugía de las fracturas dorsales con abordaje posterior ${ }^{5,12,22}$, aunque sí en la cirugía de la escoliosis ${ }^{2,17,19}$ o en la de extirpación de tumores por esta misma vía de abordaje ${ }^{20}$.

Para el tratamiento quirúrgico de las lesiones traumáticas infecciosas, neoplásicas y degenerativas de la columna dorsal el abordaje tradicional utilizado ha sido por vía transtorácica o anterolateral. Esta vía facilita una excelente exposición de los cuerpos vertebrales y de sus estructuras laterales pero requiere la violación del espacio pleural y la manipulación de pulmones y las estructuras mediastínicas, por lo que necesita la colocación de un tubo de drenaje pleural que añade morbilidad a la intervención y puede retrasar la movilización del paciente ${ }^{13}$. Una alternativa a esta vía es la posterolateral, que se ha utilizado para el tratamiento de las fracturas dorsolumbares sin este inconveniente con buenos resultados ${ }^{14}$. No hay unanimidad en la existencia de recomendaciones estrictas para seleccionar un abordaje o el otro ${ }^{10,18}$.

Tras detectar en la práctica clínica diaria la presencia de derrame pleural en algunos pacientes sometidos a fijación vertebral por vía posterior para realizar la fijación de su fractura vertebral, que en algún caso ha necesitado de la realización de toracocentesis para solucionar una insuficiencia respiratoria de tipo restrictivo que provocaba, hemos decidido hacer un estudio de cohortes prospectivo para determinar cúal es la incidencia de esta complicación en nuestros pacientes.

Nuestros resultados muestran la presencia de derrame pleural en el $75 \%$ de los pacientes intervenidos para fijación espinal dorsolumbar tras una fractura espinal con afectación y daño medular. No está clara su fisiopatología, dado que, excepto en caso de complicaciones imprevistas, no se aborda la cavidad intrapleural durante la intervención, a diferencia del abordaje anterior o anterolateral, en el que se 
mantiene un drenaje intratorácico tras la cirugía para evitar la presencia de derrame pleural postquirúrgico que complique la función respiratoria en el postoperatorio.

Los mecanismos para producir un derrame pleural son múltiples ${ }^{21}$. Probablemente los mecanismos involucrados en el derrame que se produce en los pacientes con lesión medular por fractura espinal dorsal y/o lumbar que se ven sometidos a cirugía de fijación por vía posterior sean el incremento en la circulación microvascular pleural y el empeoramiento del drenaje linfático como consecuencia de la manipulación de la pleura posterior por cercanía al área de intervención quirúrgica. El hecho de que el derrame sea exudado en todos nuestros casos analizados apoya esta teoría. Además en el caso de cirugía con una técnica similar pero con menor y más corta manipulación, como es la retirada, en vez de colocación, del material de osteosíntesis, no se acumula dicho derrame sino infrecuentemente.

En 4 de los 15 casos con derrame pleural, el 26,7\%, el volumen que llega a acumularse es de tal magnitud que se necesita su drenaje mediante un tubo de toracocentesis para solucionar la situación de insuficiencia ventilatoria restrictiva. En el restante número de casos $(9$, el 73,3\%) basta con una actitud conservadora, ya que hemos podido comprobar cómo el derrame se ha reabsorbido a los 15 días en la totalidad de los casos. Aunque en 6 de estos 9 casos hemos utilizado empíricamente tratamiento con Indometacina oral, por similitud a su indicación en el tratamiento de otro tipo de derrames de origen inflamatorio, como los que provocan la artritis reumatoide, el lupus eritematoso sistémico o el síndrome de Dressler ${ }^{21}$, sugerimos que, dada la evolución favorable de esta complicación, no se precisa tratamiento con antiinflamatorios.

\section{Conclusiones}

La presencia de derrame pleural postoperatorio es muy frecuente tras la cirugía de fijación espinal dorsal o dorsolumbar por vía posterior. Su debut es precoz, ya que se puede detectar dentro de las primeras 24 horas tras la cirugía. Es necesaria la realización de una radiografía simple de tórax a los pacientes intervenidos para el control de la presencia de derrame y la necesidad de evacuación en su caso. La mayoría de los derrames es de pequeña cantidad y son de tipo exudado, como es de esperar en derrames tras una agresión con irritación pleural. La presencia de derrame es más frecuente cuanto mayor sea la manipulación tisular en el acto quirúrgico. Sólo en algunos casos la presencia del derrame provoca insuficiencia respiratoria que obliga a su evacuación. Su historia natural tiende a su reabsorción en pocos días, por lo que el tratamiento conservador es eficaz. No creemos que sea necesaria la colocación de drenaje pleural a los postoperados, a no ser que durante el acto quirúrgico se hubiera abierto accidentalmente la hoja pleural parietal,

\section{Bibliografía}

1. Azoulay, E.: Pleural effusions in the intensive care unit. Curr Opin Pulm Med. 2003; 9: 291-297.

2. Baron, E.M., Albert, T.J.: Medical complications of surgical treatment of adult spinal deformity and how to avoid them. Spine 2006; 31 Suppl: S106-S118.

3. Bhat, A.L., Lowery, G.L.: Chylous injury following anterior spinal surgery: case reports. Eur Spine J. 1997; 6: 270-272.

4. Borges, L.F.: Surgical approaches to the thoracic and thoracolumbar spine for descompression and stabilization. In: Schmidek HH and Sweet WH eds. Operative neurosurgical techniques. WB Saunders and Co., Philadelphia; 1995: pp 1887-1893.

5. Danisa, O.A., Shaffrey, C.I., Jane, J.A., et al.: Surgical approaches for the correction of unstable thoracolumbar burst fractures: a retrospective analysis of treatment outcomes. J Neurosug 1995; 83: 977-983.

6. Fessler, R.G., Dietze, D.D.: Surgical approaches to the cervicothoracic junction. In: Schmidek HH and Sweet WH eds. Operative neurosurgical techniques. WB Saunders and Co., Philadelphia; 1995: pp 1875-1886.

7. Fletcher, D.J., Taddonio, R.F., Byrne, D.W., et al.: Incidence of acute care complications in vertebral column fracture patients with and without spinal cord injury. Spine 1995; 20: 1136-1146.

8. Fraser, R.S.: Anomalías Pleurales. En: Fraser RS, Muller NL, Colman N, Paré PD. Eds. Diagnóstico de las Enfermedades del Tórax 4a ed. Panamericana, Buenos Aires ; 2002. pp: 556-587.

9. Fraser, R.S.: Complicaciones de procedimientos terapéuticos, de biopsia y monitoreo. En: Fraser RS, Muller NL, Colman N, Paré PD. Eds. Diagnóstico de las Enfermedades del Tórax 4a ed. Panamericana, Buenos Aires ; 2002. pp: 2639-2674.

10. Hitchon, P.W., Torner, J., Eichholz, K.M., Beeler, S.N.: Comparison of anterolateral and posterior approaches in the management of throracolumbar burst fractures. J Neurosurg Spine 2006, 5: 117-125.

11. Jimenez, M.F., Roca, R., Alvarez, C., Loinaz, C., Toledo, J.: Persistent pleural effusion and post-traumatic subarachnoidal-pleural fistula. Eur J Cardiothorac Surg. 1991; 5: 554-556.

12. Knop, C., Fabian, H.F., Bastian, L., Blauth, M.: Late results of thoracolumbar fractures after posterior instrumentation and transpedicular bone grafting. Spine; 2001; 26: 88-99.

13. Kostuik, J.P.: Anterior spinal cord descompression for lesions of the thoracic and lumbar spine, techniques, new methods of internal fixation results. Spine 1983; 8: 512-531. 
14. Larson, S.J., Hoist, R.A., Hemmy, D.C., et al.: Lateral extracavitary approach to traumatic lesions of the thoracic and lumbar spine J Neurosurg 1976; 45: 628-637.

15. Loddenkemper, R., Frank, W.: Pleural Disease. In: Gibson GJ, Gedder DM, Sterk PJ, Costabel U, Corrin B. eds. Respiratory Medicine, third ed.. Elsevier, Edimburgh, 2003; pp: 1907-1937.

16. Mantel, J.K., Norman, J.R.: Respiratory complications and management of spinal cord injury. Chest 1990; 97: 1446-1452.

17. Pérez Caballero Macarrón, C., Burgos Flores, J., Martos Sanchez, I., et al.: Complicaciones médicas precoces en el postoperatorio de cirugía de escoliosis. An Pediatr (Barc) 2006; 64: 248-251.

18. Schnee, C.L., Ansell, L.V.: Selection criteria and outcome of operative approaches for thoracolumbar burst fractures with and without neurological deficit. J Neurosurg 1997; 86: 48-55.

19. Shapiro, G., Green, D.W., Fatica, N.S., Boachie-Adjei, O.: Medical complications in scoliosis surgery. Curr Opin
Pediatr. 2001; 13: 36-41.

20. Steck, J.C., Dietze, D.D., Fessler, R.G.: Posterolateral approach to intradural extramedullary thoracic tumors. J neurosurg 1994; 81: 202-205.

21. Villena Garrido, V., Ferrer Sancho, J., Hernandez Blasco, et al.: Diagnosis and treatment of pleural effusion. Arch Bronconeumol 2006; 42: 349-372.

22. Yue, J.J., Sossan, A., Selgrath, C., et al.: The treatment of unstable thoracic spine fractures with transpedicular screw instrumentation: a 3-year consecutive series. Spine 2002; 27 : 2782-2787.

Romero-Ganuza, F.J.; Marín Ruiz, M.Á.; Díez de la LastraBuigues, E.: Derrame pleural tras cirugía de fijación espinal en pacientes con lesión medular traumática. Neurocirugía 2009; 20: 39-43.

Correspondencia postal: F. Javier Romero Ganuza. UCI. Hospital Nacional de Parapléjicos Finca "La Peraleda" s/n. 45071-Toledo. 